\title{
Ultra Low Voltage Secondary and Backscatter Imaging in FE-SEM - Successes and Challenges.
}

\author{
Natasha Erdman, Vern Robertson and Masateru Shibata
}

JEOL USA Inc., 11 Dearborn Rd, Peabody, MA.

In the last decade there has been a quantum leap in the ability of the scanning electron microscope (SEM) to observe a variety of materials and biological specimens with ultra-high resolution and exceptional surface detail, in particular employing low voltage SEM. Low voltage imaging has been successfully employed as a key technique for charge control and reduction. Improvements in electron column optics towards smaller chromatic and spherical aberration coefficients, with improved ability to deal with charging specimens via precise control of the landing energy of impact electrons and electron signal detection through in-column signal filtering or signal collection angle control have opened new avenues for specimen observation [1]. In particular, these new design improvements have significantly advanced the ability to image insulating specimens with previously unattainable nanometer scale resolution [2] at landing voltages as low as 10V (Fig. 1).

In this paper we will discuss common approaches and challenges associated with ultra-low voltage imaging. The instrument employed for these studies is JSM-7800F ultra-high resolution FE-SEM that features a hybrid lens design and the ability to bias the specimen stage thus decelerating the primary beam (Gentle Beam). When beam deceleration is employed the accelerating voltage which along with lens aberrations determines the minimum probe size and thus the resolution limit is retarded by a negatively charged bias to a lower landing energy. The landing voltage $\left(E_{\text {landing }}=E_{\text {gun }}-E_{\text {bias }}\right)$ can be varied with a combination of electron source voltage and specimen bias to achieve the necessary charge balance as well as high resolution performance at ultra-low voltages. Beam deceleration also serves as a form of aberration correction [3]; the aberration coefficients (both spherical and chromatic) are reduced when the ratio $E_{\text {landing }} / E_{\text {gun }}$ is reduced for a fixed $E_{\text {gun }}$, meaning larger specimen bias enhances image resolution at ultra-low $\mathrm{kVs}$. The use of Gentle Beam function preserves all the advantages of high $\mathrm{kV}$ imaging (gun brightness, small probe size) with added advantages of reduced charging, reduced specimen contamination and improved surface detail. In addition to beam deceleration, through-the-lens detection system features an ability to precisely filter the detected signal, providing the user with an additional degree of control during the imaging. For example, Fig. 2 shows an image of Au nanoparticles on $\mathrm{TiO}_{2}$ imaged using through-the-lens detectors, employing signal filtering capability.

We will demonstrate our experiences with imaging a variety of specimens, such as zeolites, biological nanostructures, oxides, nano-structured metals and more. We will also highlight the ability to collect backscatter images at very low accelerating voltages, down to few 100 s of volts (Fig. 3). The advantages of low $\mathrm{kV}$ imaging for such techniques as cathodoluminescence imaging and voltage contrast will be highlighted. Additional methods for charge balance, such as reduced probe current and adjustment of scan speed will also be discussed.

\section{References:}

[1] D.C. Bell and N. Erdman. Low Voltage Electron Microscopy: Principles and Applications (2012)

[2] S. Asahina et al., Microscopy and Analysis, (2012) p.S12.

[3] L. Frank and I. Mullerová, Ultramicroscopy, 106 (2005) p. 28 


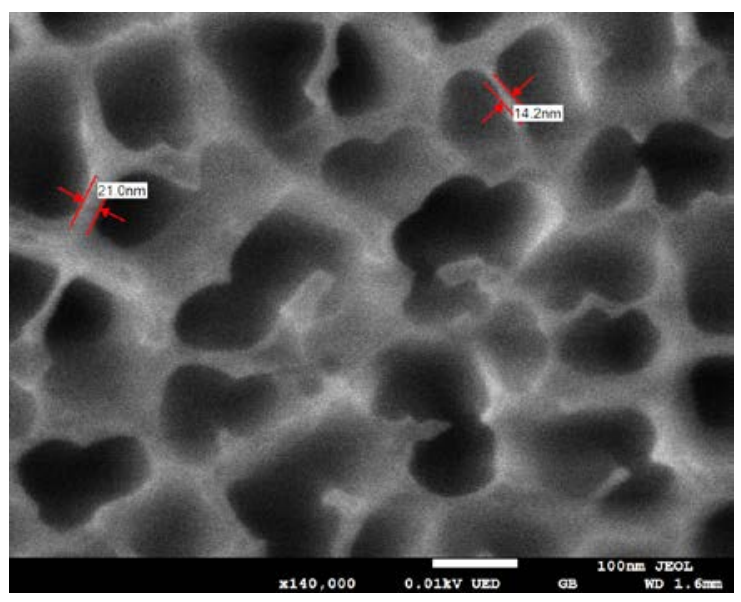

Figure 1. Anopore membrane filter imaged uncoated at 10V. Pore walls (14 and $21 \mathrm{~nm}$ ) are clearly resolved.
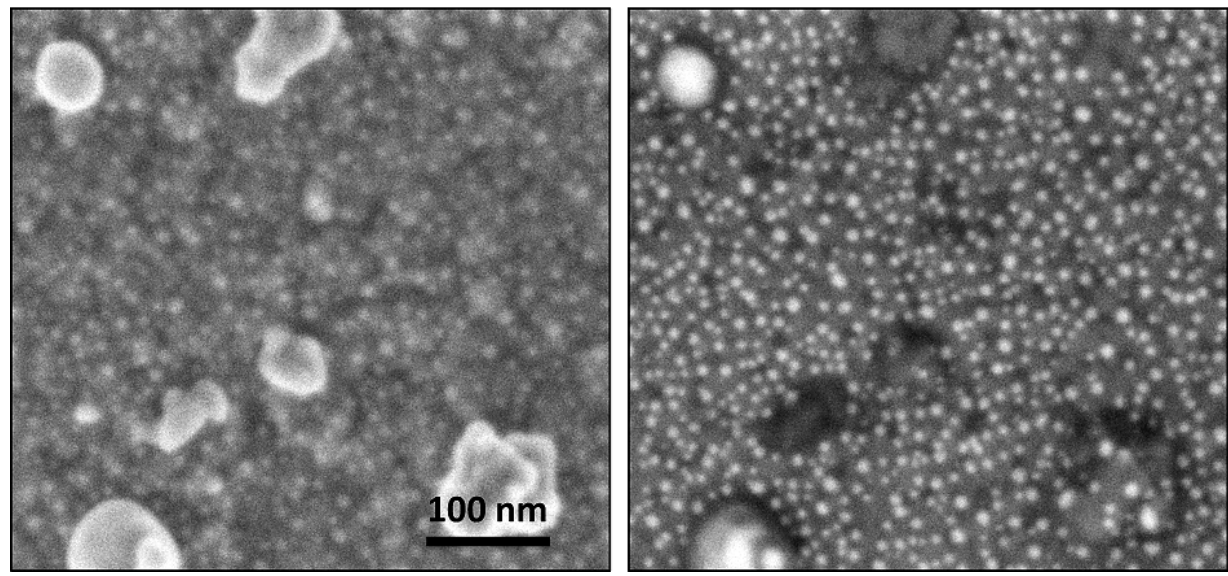

Figure 2. $\mathrm{Au}$ nanoparticles $(2-10 \mathrm{~nm})$ on $\mathrm{TiO}_{2}$ imaged with through the lens detectors at $1 \mathrm{kV}-\mathrm{SE}$ image taken with USD detector (left) and BE image taken with UED detector using -300V filtering grid bias (right).

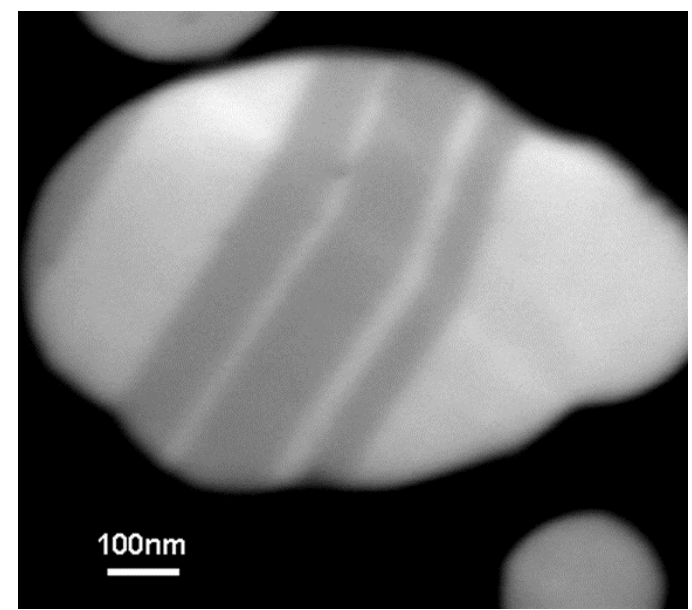

Figure 3. Au nanocrystal imaged at $500 \mathrm{~V}$, showing crystallographic orientation contrast with nanosized twins. 